\title{
Bite force measurement based on fiber Bragg grating sensor
}

Srivani Padma

Sharath Umesh

Sundarrajan Asokan

Talabattula Srinivas 


\title{
Bite force measurement based on fiber Bragg grating sensor
}

\author{
Srivani Padma, ${ }^{a, b}$ Sharath Umesh, ${ }^{a}$ Sundarrajan Asokan, ${ }^{a, c, *}$ and Talabattula Srinivas ${ }^{\mathrm{b}, \mathrm{c}}$ \\ andian Institute of Science, Department of Instrumentation and Applied Physics, Bangalore, India \\ ${ }^{b}$ Indian Institute of Science, Department of Electrical Communication Engineering, Bangalore, India \\ Indian Institute of Science, Applied Photonics Initiative, Bangalore, India
}

\begin{abstract}
The maximum level of voluntary bite force, which results from the combined action of muscle of mastication, joints, and teeth, i.e., craniomandibular structure, is considered as one of the major indicators for the functional state of the masticatory system. Measurement of voluntary bite force provides useful data for the jaw muscle function and activity along with assessment of prosthetics. This study proposes an in vivo methodology for the dynamic measurement of bite force employing a fiber Bragg grating (FBG) sensor known as bite force measurement device (BFMD). The BFMD developed is a noninvasive intraoral device, which transduces the bite force exerted at the occlusal surface into strain variations on a metal plate. These strain variations are acquired by the FBG sensor bonded over it. The BFMD developed facilitates adjustment of the distance between the biting platform, which is essential to capture the maximum voluntary bite force at three different positions of teeth, namely incisor, premolar, and molar sites. The clinically relevant bite forces are measured at incisor, molar, and premolar position and have been compared against each other. Furthermore, the bite forces measured with all subjects are segregated according to gender and also compared against each other. $\odot 2017$ Society of Photo-Optical Instrumentation Engineers (SPIE) [DOI: 10.1117/1.JBO.22.10.107002]
\end{abstract}

Keywords: bite force measurement device; fiber Bragg grating sensor; variable distance of biting platform.

Paper 170476R received Jul. 20, 2017; accepted for publication Oct. 11, 2017; published online Oct. 31, 2017.

\section{Introduction}

The force exerted by the combined action of the maxillary and mandibular tooth of the masticatory system during dental occlusion is known as the individual bite force. The bite force is an important factor in determining the functional state of the masticatory system, which comprises of jaw muscles, teeth, and joints. ${ }^{1,2}$ The measurement of individual bite force is mainly used in dentistry to understand the state of mastication and also for the evaluation of prosthetic devices along with the assessment of therapeutic procedures. Furthermore, the measurement of bite force aids in various applications of maxillofacial surgery, odontology, etc. ${ }^{3-5}$ Many factors like the positional offset of teeth and mandible, load on the periodontal tissue, age, gender, etc. influence the generated bite force. ${ }^{6}$ Apart from these biological factors, the mechanical properties of the recording system, position of the recording device in dental arch, and unilateral or bilateral measurements influence the bite force measurement. ${ }^{4}$

As the measurement of bite force plays a vital role in the dentistry, various studies have been carried out on the bite force using various devices. Most of the devices used for the bite force measurement are developed employing either electrical or electronic sensors based on resistance, capacitance, inductance, strain, piezoelectric, or thermoelectric property, which have their own inherent drawbacks; ${ }^{7-13}$ the primary being the electrical power requirement, which is not desirable for in vivo studies. The optical fiber-based bite force measurement methodologies have also been proposed, with their inherent

*Address all correspondence to: Sundarrajan Asokan, E-mail: sundarrajan .asokan@gmail.com advantages such as electrical passiveness, high sensitivity, ultrafast response, and immunity to electromagnetic interference, overcoming some of the draw backs of electrical sensors. ${ }^{14,15}$ Specifically, fiber Bragg grating (FBG) sensors are being extensively used for sensing purposes due to many advantages, which include small dimension, multiplexing capability, etc. ${ }^{16}$ There are many studies in the literature that report the use of FBG for bite force measurement either in vivo or ex vivo by monitoring pressure and temperature of dental splint, by measuring the bite force as a function of load applied, or embedding FBG in the artificial maxilla model, etc. ${ }^{17-21}$ The optical sensors are normally limited by the resolution of measurement. ${ }^{14,15}$ An optical fiber-based bite force measurement device (BFMD) with a better resolution has been reported recently, which is again limited by the fixed distance between the biting platform. ${ }^{22}$

This study proposes an intraoral BFMD with adjustable distance between biting platforms employing FBG sensor. The developed BFMD converts the bite force exerted at the occlusal surface region into strain variations on a metal plate. These strain variations on the metal plate are acquired by the FBG sensor bonded over it. The major advantage of the BFMD developed over its counterparts is the flexibility of adjusting the distance between the biting platforms depending on the position of the tooth whose individual bite force is measured. Also, the device developed facilitates the FBG sensor outside the oral cavity. The advantages associated with BFMD along with the inherent advantages of FBG sensor ${ }^{23}$ make the present BFMD an effective means for the measurement of bite force.

The bite force measurements undertaken in this study, on a substantial sample size, prove the efficacy of the developed 
BFMD. In this work, the variation of bite force at different teeth positions along the dental arch is also compared. In addition, a comparison of bite force measured with respect to gender is carried out in this study.

\section{Materials and Methods}

\subsection{Theory of Fiber Bragg Grating Sensor}

FBG is the periodic perturbation of refractive index along the core of a single-mode photosensitive optical fiber. ${ }^{24}$ The perturbation of refractive index is brought about by exposing the optical fiber core to an interference pattern from an intense UV laser light. The FBG acts as a selective mirror, which reflects a particular wavelength of light when a broadband wavelength of light is launched into the optical fiber and all other wavelengths are transmitted. ${ }^{23}$ The reflected wavelength of light is known as the Bragg wavelength $\left(\lambda_{\mathrm{B}}\right)$, which is governed by the Bragg condition given by

$\lambda_{\mathrm{B}}=2 n_{\mathrm{eff}} \Lambda$,

where $n_{\text {eff }}$ is the effective refractive index of the fiber and $\Lambda$ is the periodicity of gratings. The FBG sensors used in this work have a gauge length of $3 \mathrm{~mm}$ and are fabricated in a photosensitive germania-doped silica fiber, using the phase mask grating inscription method. ${ }^{25}$

Normally, the FBG sensor is sensitive to two parameters strain and temperature. Any external perturbation in the form of strain and temperature over the FBG sensor alters the periodicity of gratings or the effective refractive index, thereby causing a shift in the reflected Bragg wavelength. By interrogating the amount of shift in Bragg wavelength, the external perturbation can be quantified. ${ }^{26,27}$ The effect of strain and temperature on the Bragg wavelength is given by

$$
\begin{aligned}
\Delta \lambda_{B}= & \lambda_{B}\left(\left\{1-\frac{n_{\mathrm{eff}}^{2}}{2}\left[P_{12}-\nu\left(P_{11}+P_{12}\right)\right]\right\}\right. \\
& \left.\times \varepsilon+\left[\alpha+\frac{\left(\frac{\left.\mathrm{d} n_{\mathrm{eff}}\right)}{\mathrm{d} T}\right)}{n_{\mathrm{eff}}}\right] \Delta T\right),
\end{aligned}
$$

where $\alpha$ is the coefficient of thermal expansion of fiber material, $\Delta T$ is the change in temperature, ${ }^{26} P_{11}$ and $P_{12}$ are the components of the strain-optic tensor, $\nu$ is the Poisson's ratio, and $\varepsilon$ is the axial strain change. ${ }^{16,28}$ The strain sensitivity of $1.20 \mathrm{pm} / \mu \varepsilon$ approximately is reported for an FBG inscribed in a germaniadoped silica fiber, ${ }^{29}$ whereas the temperature sensitivity is of the order of $10 \mathrm{pm} /{ }^{\circ} \mathrm{C} .{ }^{27}$

In this study, the effect of temperature on FBG sensor is neglected as the experiment is carried out in a controlled environment and all the tests are carried out in a short duration (10 to $20 \mathrm{~s}$ ) during which no change in temperature is observed.

\subsection{Design and Fabrication of Bite Force Measurement Device}

The device developed consists of two rectangular rods of dimensions $100 \mathrm{~mm} \times 5 \mathrm{~mm} \times 4 \mathrm{~mm}$, which are riveted at the center with a movable joint, which makes the two rods mimic a scissor action. Square plates of dimension $10 \mathrm{~mm} \times 10 \mathrm{~mm} \times 1.5 \mathrm{~mm}$ are welded to their edges on one side and L-angle square plates are attached to the other side, such that the square plates are aligned along the same plane, as shown in Fig. 1.

The square plates on one side with hardened rubber material between them is utilized as the biting platform, and the L-angle square plates are connected using a rectangular stainless-steel plate of dimension $30 \mathrm{~mm} \times 10 \mathrm{~mm} \times 0.3 \mathrm{~mm}$. The L-angle plates comprise of grooves, through which the rectangular plate is screwed on. This facilitates to adjust the distance between the L-angle plates, which in turn will vary the distance between the biting platforms.

By the change in the position of the screw in the groove of the rectangular plate, the required specific distance between the biting platforms can be achieved. Corresponding to the distance between the biting platforms, the hardened rubber is changed in between the biting platform. An FBG sensor is bonded over the rectangular plate, which has the ability to acquire the strain variations over it. Rubber films are attached on the biting platform in order to provide cushion to the teeth applying the bite force. Together, all these components constitute to form the proposed BFMD.

The bite force applied over the biting platform makes the square plates converge. This converging action of the biting platform creates a diverging action of the L-angle square plates, which in turn exert a tensile force on the rectangular plate axially. This tensile force creates strain variations over the rectangular plate, which is acquired by the FBG sensor bonded over it.

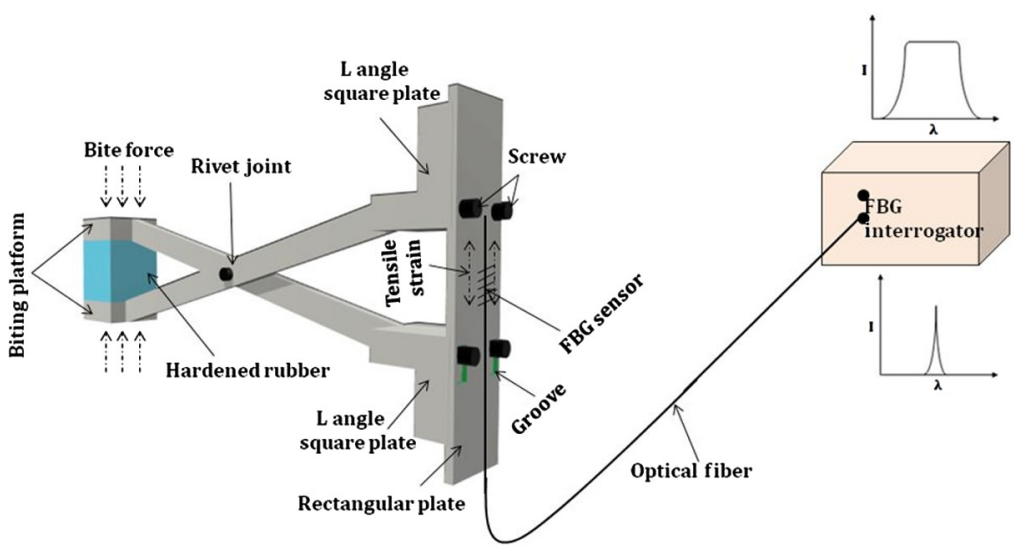

(a)

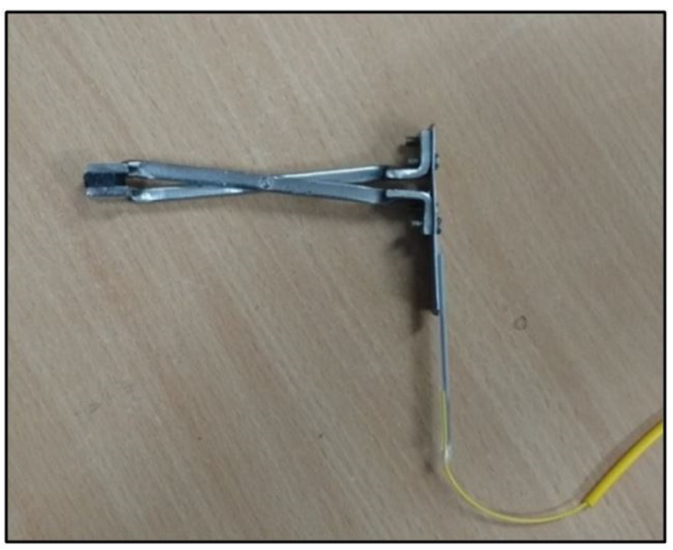

(b)

Fig. 1 (a) schematic and (b) pictorial representation of BFMD. 


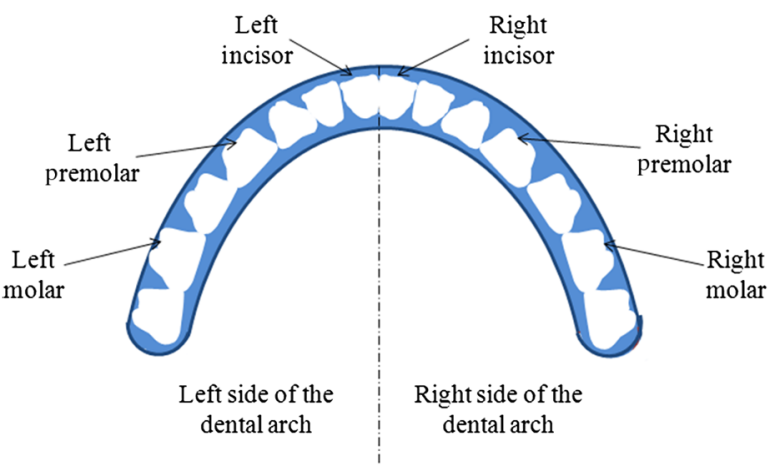

(a)

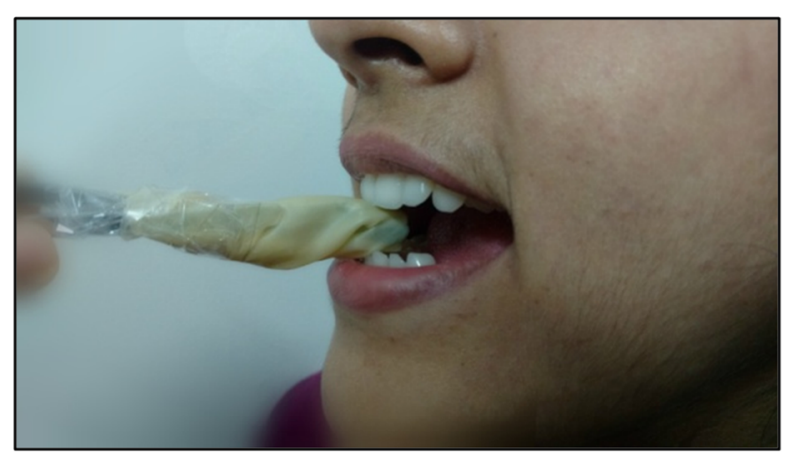

(b)

Fig. 2 (a) Dental arch representing the tooth positions chosen for the bite force measurement and (b) subject clenching the biting platform.

The magnitude of strain variation over the rectangular plate is directly dependent on the magnitude of the force applied on the biting platform. Essentially, the proposed BFMD transduces the applied bite force into strain variations, which are acquired through the FBG sensor. Any change in position of the screw on the L-angle plate, i.e., a change in the distance of the biting platform, does not affect the magnitude of strain variation generated on the rectangular plate. Therefore, the sensitivity of the proposed BFMD remains the same irrespective of the distance between the biting platforms.

\subsection{Experimental Methodology}

The experiments carried out in this study are within the guidelines of the Indian Institute of Science Human Ethics Committee and are carried out under the strict supervision of a registered dental practitioner. Twenty-two subjects (10 male and 12 female) aged between 22 and 40 years have volunteered for this study. The volunteered subjects are known to have a good dentition history without any surgery, craniofacial trauma, cuspal coverage, cast restorations, and symptoms of temporomandibular or craniocervical disorders.

Two molar, two premolars, and two incisors (symmetric tooth positions on either side of the dental arch) are selected in order to measure the individual bite force for each subject. Prior to the onset of the test, the subjects are detailed about the experimental procedure. The distance between the biting platforms is adjusted by the screw assembly on the L-angle plates depending on the position of the tooth where the bite force is to be measured. The distance between the biting platforms is fixed at 6,10 , and $14 \mathrm{~mm}$ for molar, premolar, and incisor bite force acquisition, respectively. ${ }^{4,30,31}$ Furthermore, the BFMD is wrapped in a disposable latex cover, which is meant for hygienic purpose of the subject.

Figure 2(a) shows the schematic of a dental arch representing the tooth positions chosen for bite force measurement. The BFMD is positioned at the chosen tooth location between the maxillary and mandibular jaws as shown in Fig. 2(b). The subject is requested to clench on the biting platform of the BFMD thrice in each of the chosen locations, which is transduced to strain on the stainless steel plate. The strain variation over the stainless-steel plate is acquired by the FBG sensor bonded over it. The BFMD is connected to FBG data acquisition system (SM 130-700 Micron Optics Interrogator), which has the ability to record the data with a sampling rate of $1 \mathrm{kHz}$ and with a resolution of $1 \mathrm{pm}$ shift in Bragg wavelength, which effectively converts to $0.81-\mu \varepsilon$ strain variation. The maximum individual bite force at that specific tooth position is acquired as the strain on the FBG sensor.

\subsection{Calibration}

The developed BFMD is calibrated against a micro universal testing machine (UTM) for its response of shift in wavelength for the load applied on the biting platform. The calibration test is carried out in a laboratory under controlled environment. Figure 3 shows the experimental setup for the calibration, where the load from the micro-UTM is applied on the biting platform. The distance between the biting platforms is initially maintained at $6 \mathrm{~mm}$.

The load is applied by the micro-UTM for a range of force from 0 to $800 \mathrm{~N}$, at a constant displacement of $2 \mathrm{~mm} / \mathrm{s}$, and the simultaneous response of the BFM is acquired. By comparing the data obtained from the BFMD and micro-UTM, it is observed that the strain of the BFMD increases hand-in-hand with the applied load on its biting platform (Fig. 4). Furthermore, the response of strain of BFMD is found to be in good agreement with the load applied by the micro-UTM, which is ascertained by the correlation coefficient of 0.99 obtained between them. The slope of the obtained curve is observed to be $0.91 \mu \varepsilon / \mathrm{N}$ indicating a strain of $0.91 \mu \varepsilon$ corresponding to $1 \mathrm{~N}$ force applied. As the response of BFMD is acquired with the Micron Optics Interrogator (SM 130-700), which has the ability to measure the Bragg wavelength with resolution of

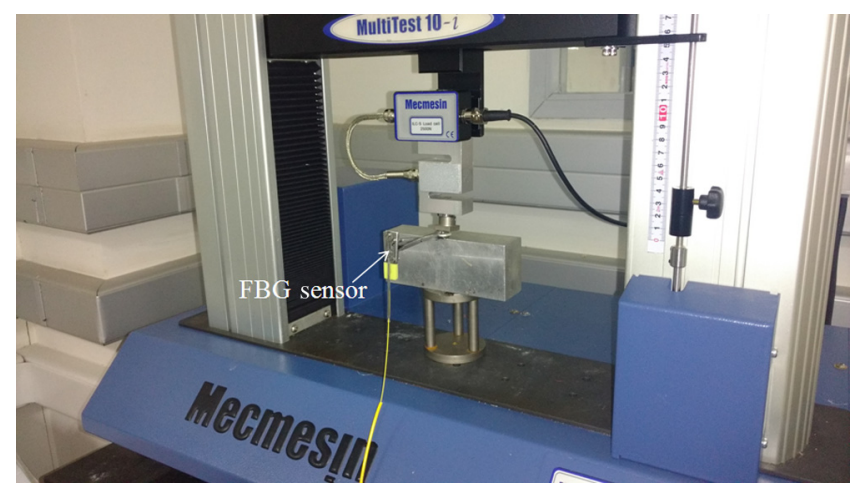

Fig. 3 Calibration setup of BFMD with micro-UTM. 


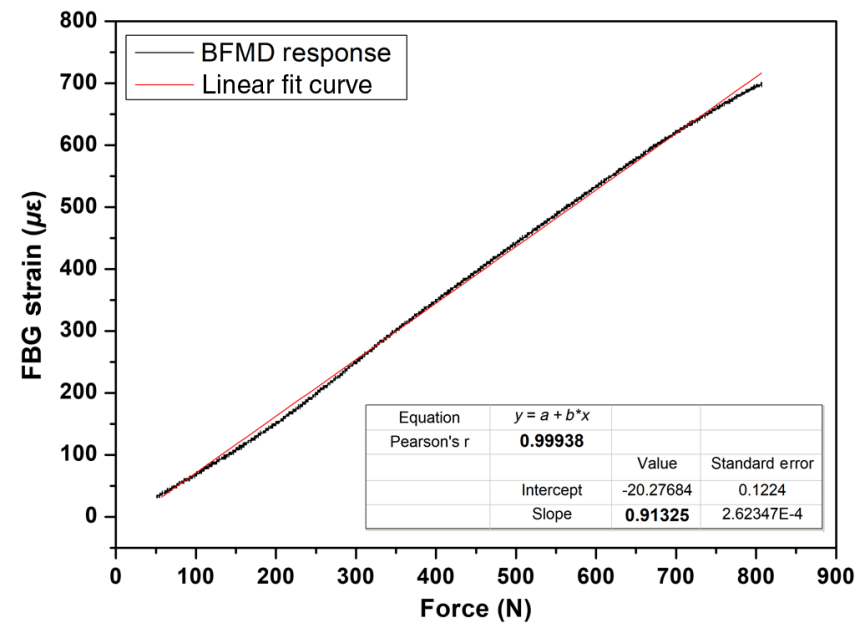

Fig. 4 BFMD response curve with respect to the load applied from micro-UTM.

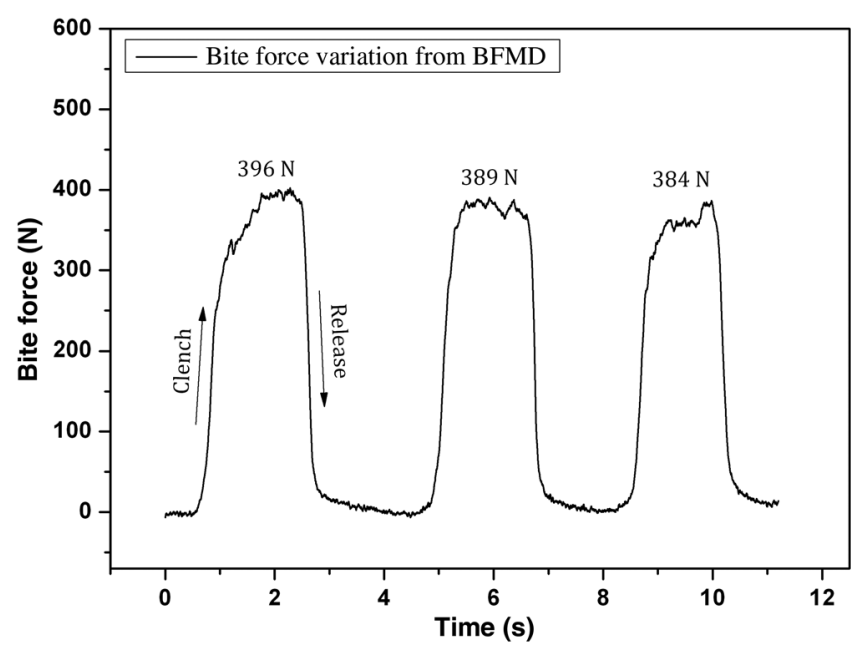

Fig. 5 Bite force recorded at premolar position for a female subject.

$1 \mathrm{pm}$ effectively $0.83 \mu \varepsilon$, and hence, the resolution of the proposed BFMD is found to be $0.90 \mathrm{~N}$.

The distance between the biting platforms is varied from 6 to $10 \mathrm{~mm}$ and $14 \mathrm{~mm}$ by the screw assembly on the L-angle plates, and similar calibration tests are carried out. It is observed that the response of the BFMD remains almost similar with varying distance between the biting platforms, which may be attributed to the tensile axial nature of the load being exerted on the stainless-steel plate.

\section{Results and Discussion}

The developed BFMD measures bite force applied on the biting platform in the form of real-time dynamic strain variations on the rectangular plate. For the illustration of the developed BFMD, the response obtained for a female subject aged 28 years at the premolar position of the left-side dental arch is selected and shown in Fig. 5.

The response of the BFMD at the premolar position during the performance of the bite force shows that the bite force increases and decreases rapidly as the BFMD is clenched and released, respectively. As the BFMD is clenched, the bite force is applied over the BFMD. The peak force recorded is considered as the maximum individual bite force at that position for the particular subject. It is also observed that the bite force varies during the clenched phase and hence the variation in the response of BFMD is observed. With the three bite force trials performed by the subject at the premolar position, the maximum individual bite forces of 396, 389, and $384 \mathrm{~N}$ are obtained. Similarly, the bite force measurement trials employing BFMD are carried out at the molar, premolar, and incisor positions on either side of the dental arch for all the 22 subjects with appropriate biting platform distances.

The maximum individual bite forces recorded for all the 22 subjects with different tooth positions are obtained and tabulated in Table 1. Furthermore, the graphical representation of the data tabulated in Table 1 in the form of box plot is shown in Figs. 6(a) and 6(b). It can be observed from Fig. 6 that the bite forces measured in molar position are maximum for both male and female subjects, followed by the premolar and incisor tooth positions.

The bite forces measured show an increasing tendency from incisor to molar teeth along the dental arch for both male and female subjects. Also, it can be observed that there is a range of measurement of bite forces of a specific tooth position in the right and left dental arch, respectively, for both male and female subjects. It is also observed that the maximum bite forces recorded for the male subjects are higher compared to the bite forces recorded for female subjects as shown in earlier studies. ${ }^{4,32,33}$ The spread in the bite forces measured with BFMD for all subjects may be attributed to the difference in the force applied by the subject during each bite due to the neuromuscular

Table 1 Bite force obtained from FBGBFR for all the 22 subjects.

\begin{tabular}{|c|c|c|c|c|c|c|c|c|c|c|c|}
\hline \multirow[b]{2}{*}{ Position } & & \multicolumn{5}{|c|}{ Bite force of female subjects $(N)$} & \multicolumn{5}{|c|}{ Bite force of male subjects $(N)$} \\
\hline & & Mean & Max & Min & SD & Range & Mean & Max & Min & SD & Range \\
\hline \multirow[t]{3}{*}{ Left } & Molar & 570 & 660 & 475 & 60 & 185 & 654 & 751 & 543 & 64 & 208 \\
\hline & Premolar & 332 & 375 & 275 & 28 & 100 & 389 & 454 & 331 & 38 & 123 \\
\hline & Incisor & 205 & 242 & 182 & 19 & 60 & 289 & 339 & 242 & 26 & 97 \\
\hline \multirow[t]{3}{*}{ Right } & Molar & 568 & 645 & 497 & 57 & 148 & 665 & 774 & 527 & 65 & 247 \\
\hline & Premolar & 345 & 415 & 269 & 39 & 146 & 401 & 475 & 348 & 33 & 127 \\
\hline & Incisor & 223 & 258 & 190 & 20 & 68 & 295 & 350 & 235 & 37 & 115 \\
\hline
\end{tabular}




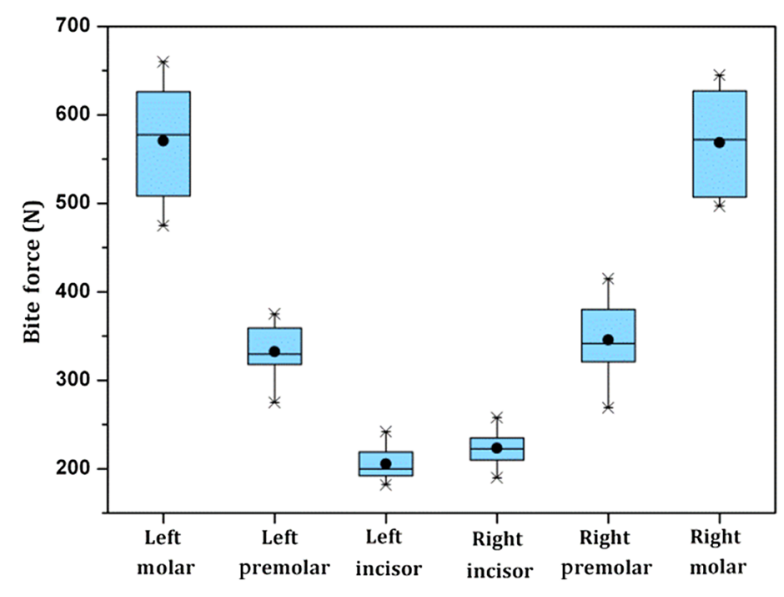

(a)

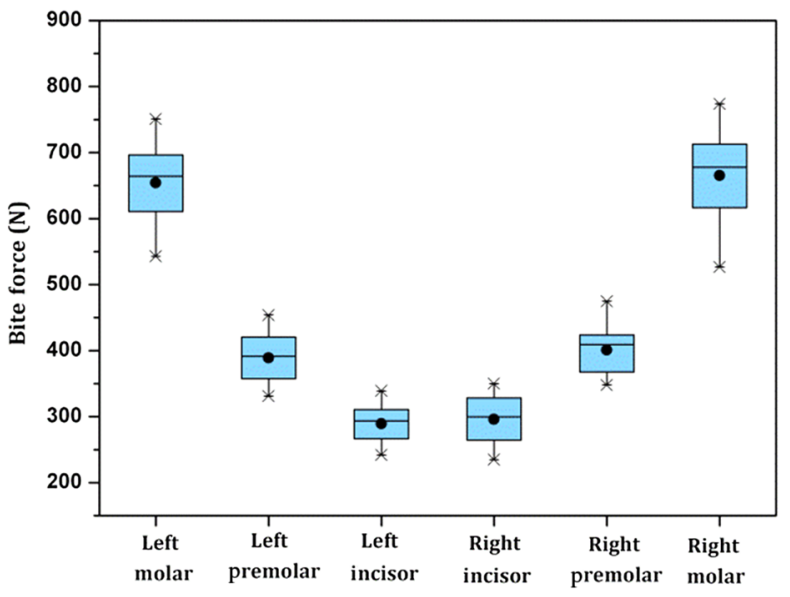

(b)

Fig. 6 Bite force measured from (a) female subjects and (b) male subjects.

reaction of the subject biting over a hard surface; irregular movements of the subject and also the positional offset of the BFMD during the tests conducted can lead to the observed spread.

The obtained results prove the efficacy of the BFMD in its ability to acquire the individual bite force. The obtained bite forces in this study are within the range of clinically relevant bite force levels. The response of the BFMD is found to be linear to the applied force on the biting platform and hence the proposed BFMD offers a noncomplex bite force system. This study also reiterates the established fact that the bite force varies according to the gender as well as the position of the tooth along the dental occlusion.

The benefits of BFMD are its ability to vary the biting platform distance, electrical passiveness, and the position of the FBG sensors outside the oral cavity. These benefits along with the inherent advantages of the FBG sensor such as small dimensions, chemical inertness, and immunity to EMI make the developed BFMD as one of the better methods to acquire the clinically relevant bite force. Furthermore, this study can be extended to find the variation of bite forces with age and also to evaluate the treatment of prosthetic devices.

\section{Conclusion}

This study proposes a real-time dynamic BFMD based on the FBG sensor, namely BFMD. The developed device has been calibrated against a micro-UTM for a load of 0 to $800 \mathrm{~N}$ over the biting platform, and a resolution of $1 \mathrm{~N}$ has been achieved for the BFMD developed.

Studies carried out using the BFMD reveal that the bite forces measured for both male and female subjects exhibit an increasing tendency from incisor to molar position along the dental arch and the maximum bite forces measured in male subjects are higher than those in the female subjects. It is also observed that the bite forces measured are symmetrical on either side of the dental arch, a specific tooth position in both male and female subjects, as obtained in earlier studies. ${ }^{22}$ This study proves the efficacy of the BFMD developed for clinically relevant measurements.

\section{Disclosures}

The authors declare that there is no conflict of interest.

\section{References}

1. M. Bakke, "Bite force and occlusion," in Seminars in Orthodontics, Vol. 12, pp. 120-126, Elsevier Inc. (2006).

2. J. Ahlgren and B. Öwall, "Muscular activity and chewing force: a polygraphic study of human mandibular movements," Arch. Oral Biol. 15(4), 271-280 (1970).

3. C. P. Fernandes et al., "A novel sensor for bite force determinations," Dent. Mater. 19, 118-126 (2003).

4. D. Koc, A. Dogan, and B. Bek, "Bite force and influential factors on bite force measurements: a literature review," Eur. J. Dent. 4, 223-232 (2010).

5. A. D. Lantada et al., "Novel system for bite-force sensing and monitoring based on magnetic near field communication," Sensors 12(9), 11544-11558 (2012).

6. K. Ando et al., "Bite force measurement system using pressure-sensitive sheet and silicone impression material," Dent. Mater. 28(2), 212-218 (2009).

7. J. F. Bates, G. D. Stafford, and A. Harrison, "Masticatory function-a review of the literature. 1. The form of the masticatory cycle," J. Oral Rehabil. 2(3), 281-301 (1975).

8. C. Hagberg, "Assessment of bite force: a review," J. Craniomandibular Disord. 1(3), 162-169 (1987).

9. A. Waltimo and M. Könönen, "A novel bite force recorder and maximal isometric bite force values for healthy young adults," Scand. J. Dent. Res. 101(3), 171-175 (1993).

10. M. Bakke et al., "Ultrasound image of human masseter muscle related to bite force, electromyography, facial morphology, and occlusal factors," Scand. J. Dent. Res. 100, 164-171 (1992).

11. W. N. Williams et al., "The influence of sensory impairment of the temporomandibular joints and central incisors on bite force discrimination," J. Craniomandibular Pract. 2, 119-124 (1984).

12. P. O. Glantz and G. D. Stafford, "Bite forces and functional loading levels in maxillary complete dentures," Dent. Mater. 1, 66-70 (1985).

13. D. V. Steenberghe and J. H. De Vries, "The development of a maximum clenching force between two antagonistic teeth," J. Periodontal Res. 13, 91-97 (1978).

14. P. Roriz et al., "From conventional sensors to fibre optic sensors for strain and force measurements in biomechanics applications: a review," J. Biomech. 47, 1251-1261 (2014).

15. H. K. Kopola et al., "Instrument for measuring human biting force," Proc. SPIE 2331, 149-155 (1995).

16. R. Kashyap, Fiber Bragg Gratings, Academic Press, San Diego (1999).

17. S. C. Tjin et al., "Recording compliance of dental splint use in obstructive sleep apnea patients by force and temperature modeling," Med. Biol. Eng. Comput. 39, 182-184 (2001).

18. M. S. Milczewski et al., "Measuring orthodontic forces with HiBi FBG sensors," in Proc. of 18th Optical Fiber Sensors Conf., Cancun, pp. 23 27 (2006).

19. M. S. Milczewski et al., "Sensitivity of silica and polymer microstructured fibres to transversal pressure," Glass Technol. 50, 211-213 (2009). 
20. M. S. Milczewski et al., "Force monitoring in a maxilla model and dentition using optical fiber Bragg gratings," Sensors 12, 11957-11965 (2012).

21. A. P. G. O. Franco et al., "Measurement of mandibular movements in parafunctional patient using occlusal splint with Bragg gratings: pilot study," Proc. SPIE 9916, 991618 (2016).

22. S. Umesh et al., "Fiber Bragg grating based bite force measurement," J. Biomech. 49, 2877-2881 (2016).

23. A. Othonos, "Fiber Bragg gratings," Rev. Sci. Instrum. 68(12), 4309_ 4341 (1997).

24. W. W. Morey, G. Meltz, and W. H. Glenn, "Fiber Bragg grating sensors," Proc. SPIE 1169, 98-107 (1989).

25. K. O. Hill et al., "Bragg gratings fabricated in monomode photosensitive optical fiber by UV exposure thorough a phase-mask," Appl. Phys. Lett. 62, 1035-1037 (1993).

26. A. D. Kersey et al, "Fiber grating sensors," J. Lightwave Technol. 15 1442-1462 (1997).

27. A. Othonos and K. Kalli, Fiber Bragg Gratings Fundamentals and Applications in Telecommunications and Sensing, Artech House, Boston (1999).
28. B. A. Tahir, J. Ali, and R. A. Rahman, "Fabrication of fiber grating by phase mask and its sensing application," J. Optoelectron. Adv. Mater. 8(4), 1604-1609 (2006).

29. S. M. Melle et al, "A Bragg grating-tuned fibre laser strain sensor system," IEEE Photonics Technol. Lett. 5, 263-266 (1993).

30. T. Shinogaya et al., "Bite force and occlusal load in healthy young subjects-a methodological study," Eur. J. Prosthodontics Restor. Dent. 8, 11-15 (2000)

31. B. R. Mackenna and K. S. Türker, "Jaw separation and maximum incising force," J. Prosthetic Dent. 49, 726-730 (1983).

32. S. Varga et al., "Maximum voluntary molar bite force in subjects with normal occlusion," Eur. J. Orthod. 33, 427-433 (2011).

33. D. Koc, A. Dogan, and B. Bek, "Effect of gender, facial dimensions, body mass index and type of functional occlusion on bite force," J. Appl. Oral Sci. 19, 274-279 (2011).

Biographies for the authors are not available. 